Masayuki Itô

Nagoya Math. J.

Vol. 53 (1974), 115-126

\title{
SUR LA FAMILLE SOUS-ORDONNÉE AU NOYAU DE CONVOLUTION DE HUNT II
}

\author{
MASAYUKI ITÔ
}

\section{Introduction et préliminaires}

Dans toute la suite $X$ désignera un groupe abélien localement compact et dénombrable à l'infini; $\xi$ sera sa mesure de Haar. Un noyau de convolution $N$ sur $X$ signifie une mesure de Radon positive dans $X$, et, pour une mesure de Radon réelle $\mu$ dans $X, N * \mu$ s'appelle le $N$ potentiel de $\mu$ dès que cette convolution a un sens. En particulier, s'il est absolument continu par rapport à $\xi$, sa densité s'écrira $N \mu$. Pour une fonction $f$ localement $\xi$-sommable dans $X$, on notera $N * f$ et $N f$ au lieu de $N *(f \xi)$ et $N(f \xi)$ dès que ceux sont définis.

Un noyau de convolution de Hunt $N$ sur $X$ est, par définition, un noyau de convolution sur $X$ de la forme

$$
N=\int_{0}^{\infty} \alpha_{t} d t
$$

où $\left(\alpha_{t}\right)_{t \geqq 0}$ est un semi-groupe vaguement continu de mesures de Radon positives $\neq 0$ dans $X$; c'est-à-dire, $\alpha_{0}=$ mesure de Dirac, $\alpha_{t} * \alpha_{s}=\alpha_{t+s}$ $\left({ }^{\forall} \geqq 0, \forall_{s} \geqq 0\right.$ ) et l'application $t \rightarrow \alpha_{t}$ est vaguement continue. Dans ce cas, ce semi-groupe est uniquement déterminé (cf. [2]), qui s'appelle le semi-group associé au noyau $N . \quad N$ est borné ${ }^{1)}$ (cela est équivalent au principe complet du maximum pour $N$ ) si et seulement si, quel que soit $t \geqq 0, \int d \alpha_{t} \leqq 1$. Dan ce cas, il existe une fonction définie-négative $\psi$ sur le groupe dual $\hat{X}$ de $X$, et une seule telle que, quel que soit $t \geqq 0, \hat{\alpha}_{t}=$ $\exp (-t \psi)$ (cf. par exemple, [4] $)^{2)}$, où la signe ^ représente la transfor-

Received December 11, 1972.

1) Un noyau de convolution $N$ sur $X$ est dit borné si, quelle que soit $f$ de $C_{K}(X)$, $N * f$ est bornée sur $X$.

2) Une fonction $\psi$ complexe et continue dans $\hat{X}$ est dite définie-négative si $\psi(\hat{0}) \geqq 0$, $\psi(-\hat{x})=\hat{\psi(\hat{x})}(\forall \hat{x} \in \hat{X})$ et quels que soient $n$ un entier $>0,\left(\hat{x}_{i}\right)_{i=1}^{n}$ une famille de points de $\hat{X}$ et $\left(c_{i}\right)_{i=1}^{n}$ une famille de nombres complexes avec $\sum_{i=1}^{n} c_{i}=0$,

$$
\sum_{i=1}^{n} \sum_{j=1}^{n} \psi\left(\hat{x}_{i}-\hat{x}_{j}\right) c_{i} \overline{c_{j}} \leqq 0
$$


mation de Fourier. On a symboliquement $\hat{N}=1 / \psi$, et $\psi$ s'appelle la fonction définie-négative associée au noyau $N$. Sous une condition additionnelle, un noyau de convolution de Hunt est un noyau de convolution $N$ qui satisfait au principe de domination; c'est-à-dire, quelles que soient $f$ et $g$ de $C_{K}^{+}(X), N * f \leqq N * g$ partout sur $X$ dès que $N * f \leqq N * g$ sur le support de $f$, supp (f) (cf. [8]). On note ici $C_{K}(X)$ et $C_{K}^{+}(X)$ l'espace vectoriel topologique usuel des fonctions finies et continues dans $X$ à support compact et son sous-ensemble des fonctions non-négatives, respectivement.

Dans l'article précédent [9], nous avons montré le théorème suivant:

Soit $\kappa$ un noyau de convolution borné sur la droite réelle $\boldsymbol{R}$ porté par $\boldsymbol{R}^{+}=\{t \in \boldsymbol{R} ; t \geqq 0\}$. Pour que, quels que soient $X$ un groupe abélien localement compact et dénombrable à l'infini, et $N=\int_{0}^{\infty} \alpha_{t} d t$ un noyau de convolution de Hunt sur $X, N_{(\kappa)}=\int \alpha_{t} d \kappa(t)$ satisfasse au principe de domination, il faut et il suffit que $\kappa$ satisfasse au principe de domination.

Dans cette note, on obtiendra d'abord le théorème suivant:

Soient $\kappa$ un noyau de convolution non-zéro sur $\boldsymbol{R}$ porté par $\boldsymbol{R}^{+}$et $N=$ $\int_{0}^{\infty} \alpha_{t} d t$ un noyau de convolution de Hunt sur $X$. Si $\kappa$ satisfait au principe de domination et si $N_{(k)}=\int \alpha_{t} d \kappa(t)$ a un sens, alors $N_{(\kappa)}$ est un noyau de convolution de Hunt. Dans ce cas, le semi-groupe associé au noyau $N_{(\kappa)}$ est explicitement représenté.

Sous une condition additionnelle pour $X$, on obtiendra ensuite l'équivalence suivante: Soit $\kappa$ un noyau de convolution sur $\boldsymbol{R}$ porté par $\boldsymbol{R}^{+}$; alors les deux énoncés suivants sont équivalents.

(1) Quel que soit $N=\int_{0}^{\infty} \alpha_{t} d t$ un noyau de convolution de Hunt sur $X, N_{(\kappa)}$ a un sens et il est un noyau de convolution de Hunt sur $X$.

(2) $\kappa$ est un noyau de convolution de Hunt borné sur $\boldsymbol{R}$.

Soit $H_{b}\left(\boldsymbol{R}^{+}\right)$la totalité des noyaux convolution de Hunt bornés sur $\boldsymbol{R}$ portés par $\boldsymbol{R}^{+}$. Pour un noyau de convolution de Hunt $N=\int_{0}^{\infty} \alpha_{t} d t$ sur $X$, on appelle

$$
H(N ; X)=\left\{N_{(\kappa)}=\int \alpha_{t} d \kappa(t) ; \kappa \in H_{b}\left(\boldsymbol{R}^{+}\right)\right\}
$$

la famille sous-ordonnée au noyau $N$ (cf. [9]). D’après la présente équivalence, cette terminologie est plus justifiée. 
Dans le cas où $N$ est borné, la fonction définie-négative associée à un élément de $H(N ; X)$ sera écrite explicitement au moyen de la fonction définie-négative associée au noyau $N$ et d'une fonction de Bernstein (voir le théorème 3 ).

Nous discuterons finalement une caractérisation pour qu'un noyau de convolution sur l'espace euclidien $\boldsymbol{R}^{n}(n \geqq 3)$ appartienne à la famille sous-ordonnée au noyau newtonien sur $\boldsymbol{R}^{n}$.

\section{La famille sous-ordonnée au noyau de convolution de Hunt}

Commençons d'abord avec la définition d'une résolvante. Une famille $\left(N_{p}\right)_{p>0}$ de noyaux de convolution sur $X$ est, par définition, une résolvante si l'on a

$$
N_{p}-N_{q}=(q-p) N_{p} * N_{q} \quad\left({ }^{\forall} p>0,{ }^{\forall} q>0\right) .
$$

Dans ce cas, il est évident que l'application $(0,+\infty) \ni p \rightarrow N_{p}$ est vaguement continue. Si, pour un noyau de convolution $N$ sur $X$, il existe une résolvante $\left(N_{p}\right)_{p>0}$ telle que $\lim _{p \rightarrow 0} N_{p}=N$ (au sens de la topologie vague), alors elle est uniquement déterminée et $\left(N_{p}\right)_{p \geqq 0}$ s'appelle la résolvante associée au noyau $N$, où $N_{0}=N$ (cf. [2]).

Soient $N$ et $\left(N_{p}\right)_{p \geqq 0}$ respectivement un noyau de convolution de Hunt sur $X$ et la résolvante associée au noyau $N$. Alors, quelle que soit $\lambda$ une mesure de Radon positive dans $\boldsymbol{R}^{+}, \int N_{p} d \lambda(p)$ appartient à la famille sous-ordonnée au noyau $N$ dès que cette intégrale a un sens. Il est connu qu'il existe la famille $\left(N^{\alpha}\right)_{0 \leqq \alpha \leqq 1}$ des puissances fractionnaires de $N$ (cf. par exemple, [10]). Pour une mesure de Radon positive $\nu$ quelconque sur [0,1], $\int N^{\alpha} d \nu(\alpha)$ appartient aussi à $H(N ; X)$, car il existe une autre mesure de Radon positive $\lambda$ dans $\boldsymbol{R}^{+}$telle que $\int N^{\alpha} d \nu(\alpha)=\int N_{p} d \lambda(p)$ (cf. par exemple, [10]). La proposition suivante est déjà presque connue dans $[8]$.

Proposition 1. Soit $N$ un noyau de convolution non-zéro sur $X$. Pour que $N$ soit un noyau de convolution de Hunt sur $X$, il faut et $i l$ suffit qu'il existe la résolvante associée au noyau $N$ et que $N$ soit nonpériodique $e^{3)}$.

3) $N$ est dit périodique à un point $x$ si $N=N * \varepsilon_{x}$, où $\varepsilon_{x}$ est la mesure de Dirac au point $x$. Si, quel que soit $x \neq 0$ de $X, N \neq N * \varepsilon_{x}$, alors $N$ est dit non-périodique. 
En effet, la condition est évidemment nécessaire. S'il existe la résolvante associée au noyau $N$, alors, pour un voisinage compact $V$ de l'origine, il existe une mesure de Radon positive $\varepsilon_{C V}^{\prime}$ dans $X$ portée par $\overline{C V}$ telle que $N \geqq N * \varepsilon_{C V}^{\prime}$ dans $X$ et $N=N * \varepsilon_{C V}^{\prime}$ dans $C V^{4)}$ (cf. le lemme 3 dans [8]), et on a

$$
\lim _{V \uparrow X} N * \varepsilon_{C V}^{\prime}=0
$$

(au sens de la topologie vague) (cf. le lemme 5 dans [8]). Si, quel que soit $V$ un voisinage compact de l'origine, $N \neq N * \varepsilon_{C V}^{\prime}$, alors notre proposition peut être montrée de la même manière que dans la proposition 3 dans [8]. Supposons donc qu'il existe un voisinage compact $V$ de l'origine telle que $N=N * \varepsilon_{C V}^{\prime}$ dans $X$. On a alors, quel que soit $W$ un voisinage compact de l'origine, $N * \varepsilon_{C W}^{\prime}=\left(N * \varepsilon_{C W}^{\prime}\right) * \varepsilon_{C V}^{\prime}$ dans $X$. Il existe évidemment un autre voisinage compact $W$ de l'origine tel que $N \neq N * \varepsilon_{C W}^{\prime}$, car $N \neq 0$. $N-N * \varepsilon_{C W}^{\prime}$ étant une mesure de Radon positive dans $X$ à support compact et ayant

$$
\left(N-N * \varepsilon_{C W}^{\prime}\right)=\left(N-N * \varepsilon_{C W}^{\prime}\right) * \varepsilon_{C V}^{\prime}
$$

dans $X$, on obtient que $\int d \varepsilon_{C V}^{\prime}=1$ et $N-N * \varepsilon_{C W}^{\prime}$ est périodique à tout le point de $\operatorname{supp}\left(\varepsilon_{C V}^{\prime}\right)$ (cf. [1]). Faisant $W \uparrow X$, on obtient que $N$ est périodique à tout le point de $\operatorname{supp}\left(\varepsilon_{C V}^{\prime}\right)$, d'où cela est en contradiction avec l'hypothèse que $N$ est non-périodique.

COROLlaIRE 1. Soit $\kappa$ un noyau de convolution non-zéro sur $\boldsymbol{R}$ porté par $\boldsymbol{R}^{+}$. Alors pour que $\kappa$ soit un noyau de convolution de Hunt sur $\boldsymbol{R}$, il faut et il suffit que $\kappa$ satisfasse au principe de domination.

En effet, il est évident que la condition est nécessaire, et on montrera son inverse. S'il existe un point $(\neq 0)$ auquel $\kappa$ est périodique, alors on a $\operatorname{supp}(\kappa) \nexists 0$, mais cela est en contradiction avec $\kappa \neq 0$ et le principe de domination pour $\kappa$, d'où $\kappa$ est non-périodique. Pour une fonction $f \mathrm{de}$ $C_{K}(R)$, la fonction $\kappa * f(x) \kappa * f(-x)$ est à support compact, et donc $\kappa * \kappa$ a un sens. On a, pour deux fonctions $f, g$ non-négatives, mesurables et bornées dans $\boldsymbol{R}$ à support compact, $\kappa f \leqq \kappa g$ presque partout sur $\boldsymbol{R}$ dès que $\kappa f \leqq \kappa g$ presque partout sur $\{x \in R ; f(x)>0\}$, qui résulte de l'équivalence des deux sortes de principes de domination (cf. [7]). Les

\footnotetext{
4) Cette mesure s'appelle une mesure balayée de $\varepsilon$ sur $C V$ relativement au noyau $N$.
} 
présents deux énoncés déduisent l'existence de la résolvante associée au noyau $\kappa$ (cf. [6] ou [10]). D'après la proposition, $\kappa$ est un noyau de convolution de Hunt sur $\boldsymbol{R}$.

THÉORÈme 1. Soient $N=\int_{0}^{\infty} \alpha_{t} d t$ un noyau de convolution de Hunt sur $X$ et $\kappa$ un noyau de convolution non-zéro sur $\boldsymbol{R}$ porté par $\boldsymbol{R}^{+}$satisfaisant au principe de domination. Alors $N_{(\kappa)}=\int \alpha_{t} d \kappa(t)$ est un noyau de convolution de Hunt sur $X$ dès que l'intégrale $\int \alpha_{t} d \kappa(t)$ a un sens.

Démonstration. D'après le présent corollaire, il existe la résolvante $\left(\kappa_{p}\right)_{p \geqq 0}$ associée au noyau $\kappa$. D'après $\kappa_{p} \leqq \kappa$,

$$
N_{\left(\kappa_{p}\right)}=\int \alpha_{t} d \kappa_{p}(t)
$$

a un sens pour tout $p \geqq 0$. On a, quels que soient $p \geqq 0$ et $q>0$,

$$
\begin{aligned}
N_{\left(\kappa_{p}\right)}-N_{\left(\kappa_{q}\right)} & =\int \alpha_{t} d\left(\kappa_{p}-\kappa_{q}\right)(t)=(q-p) \int \alpha_{t} d \kappa_{p} * \kappa_{q}(t) \\
& =(q-p) \iint \alpha_{t+s} d \kappa_{p}(t) d \kappa_{q}(s)=(q-p) \iint \alpha_{t} * \alpha_{s} d \kappa_{p}(t) d \kappa_{q}(s) \\
& =(q-p) N_{\left(\kappa_{p}\right)} * N_{\left(\kappa_{q}\right)},
\end{aligned}
$$

et par suite $\left(N_{\left(\kappa_{p}\right)}\right)_{p \geqq 0}$ est une résolvante et $N_{\left(\kappa_{p}\right)} \leqq N_{(\kappa)}(p \geqq 0)$. La famille $\left(\kappa_{p}\right)_{p>0}$ converge d'une manière croissante vers $\kappa$ avec $p \downarrow 0$, et par suite $\left(N_{\left(x_{p}\right)}\right)_{p>0}$ converge aussi d'une manière croissante vers $N_{(x)}$ avec $p \downarrow 0$, d'où il existe la résolvante associée au noyau $N_{(s)}$. Montrons ensuite que $N_{(\kappa)}$ est non-périodique. $\kappa$ étant un noyau de convolution de Hunt sur $\boldsymbol{R}$, pour un entier $n>0$ quelconque, il existe une mesure de Radon positive $\varepsilon_{n}^{\prime}$ dans $R$ portée par $[1 / n,+\infty)$ telle que $\kappa \geqq \kappa * \varepsilon_{n}^{\prime}$ dans $R, \kappa=\kappa * \varepsilon_{n}^{\prime}$ dans $(-\infty, 0) \cup(1 / n,+\infty)$ et $\kappa \neq \kappa * \varepsilon_{n}^{\prime}$. Soit $x$ un point quelconque auquel $N_{(\kappa)}$ est périodique; alors, d'après l'égalité

$$
N_{\left(x-\kappa * \varepsilon_{n}^{\prime}\right)}=N_{(\kappa)} *\left(\varepsilon-N_{\left(\varepsilon_{n}^{\prime}\right)}\right)
$$

$N_{\left(x-\kappa * \varepsilon_{n}^{\prime}\right)}$ est aussi périodique au point $x$, où $\varepsilon$ est la mesure de Dirac à l'origine dans $X$. Posons $1 / a_{n}=\int d\left(\kappa-\kappa * \varepsilon_{n}^{\prime}\right)>0$; alors $\operatorname{supp}\left(a_{n}\left(\kappa-\kappa * \varepsilon_{n}^{\prime}\right)\right)$ $\subset[0,1]\left({ }^{\forall} n \geqq 1\right)$ et la suite $\left(a_{n}\left(\kappa-\kappa * \varepsilon_{n}^{\prime}\right)\right)_{n=1}^{\infty}$ converge vaguement vers la mesure de Dirac dans $R$ avec $n \rightarrow+\infty$. Donc $\left(a_{n} N_{\left(\kappa-\kappa * \varepsilon_{n}^{\prime}\right)}\right)_{n=1}^{\infty}$ converge vaguement vers $\varepsilon$ avec $n \rightarrow+\infty$, d'où $x=0$. Il est non-périodique, et 
par suite $N_{(\kappa)}$ est un noyau de convolution de Hunt sur $X$. La démonstration est ainsi complète.

Proposition 2. Soient $N=\int_{0}^{\infty} \alpha_{t} d t$ un noyau de convolution de Hunt sur $X$ symétrique par rapport à l'origine et avec $\int d N=+\infty$, et $\kappa$ un noyau de convolution de Hunt sur $\boldsymbol{R}$ porté par $\boldsymbol{R}^{+}$; alors pour que $N_{(\boldsymbol{x})}$ ait un sens, il faut et il suffit que $\kappa$ satisfasse au principe complet $d u$ maximum ${ }^{5}$.

Démonstration. La condition est évidemment suffisante (cf. le lemme 1 dans [9]). Montrons que la condition est nécessaire. On peut supposer $\kappa \neq 0$, et donc, d'après le présent théorème, $N_{(x)}$ est un noyau de convolution de Hunt sur $X$ et $\left(N_{\left(\kappa_{p}\right)}\right)_{p \geqq 0}$ est la résolvante associée au noyau $N_{(\kappa)}$, où $\left(\kappa_{p}\right)_{p \geqq 0}$ est la résolvante associée au noyau $\kappa . N$ étant symétrique par rapport à l'origine, quel que soit $t \geqq 0, \alpha_{t}$ l'est aussi. Donc $N_{(\kappa)}$ est symétrique par rapport à l'origine, et par suite, quel que soit $p>0$,

$$
p \int d N_{\left(\kappa_{p}\right)}=\iint d \alpha_{t} d\left(p \kappa_{p}\right)(t) \leqq 1
$$

(cf. par exemple, [7]). Ayant $\int d N=+\infty$, on a, quel que soit $t \geqq 0$, $\int d \alpha_{t}=1$, et par suite $p \int d \kappa_{p} \leqq 1\left({ }^{\forall} p>0\right)$, d'où $\kappa$ satisfait au principe complet du maximum. La démonstration est ainsi complète.

Pour montrer l'inverse du théorème 1, on préparera d'abord le lemme suivant:

LEMme 1. Pour qu'il existe un noyau de convolution de Hunt sur $X$ associé auquel la fonction définie-négative est purement imaginaire, il faut et il suffit qu'il existe un espace vectoriel topologique sur $\boldsymbol{R}$ appartenant à $X$ et homéomorphe avec $\boldsymbol{R}$.

En effet, supposons qu'il existe un noyau de convolution de Hunt $N$ sur $X$ associé auquel la fonction définie-négative $\psi$ est purement imaginaire. Soit $\left(\alpha_{t}\right)_{t \geqq 0}$ le semi-groupe associé au noyau $N$; alors la transformée de Fourier de $\alpha_{t} * \check{\alpha}_{t}$ est égale à 1 pour tout $t \geqq 0$, où $\check{\alpha}_{t}$. est une mesure de Radon positive dans $X$ symétrisant avec $\alpha_{t}$ par rapport à l'origine. On a donc $a_{t} * \check{\alpha}_{t}=\varepsilon$, et par suite il existe un point $x_{t}$ de $X$

5) Cela signifie que, quelles que soient $f, g$ de $C_{K}^{+}(\boldsymbol{R}), \kappa * f \leqq \kappa * g+1$ sur $\boldsymbol{R}$ dès que $\kappa * f \leqq \kappa * g+1$ sur $\operatorname{supp}(f)$. 
tel que $\alpha_{t}=\varepsilon_{x_{t}}$. On pose $E=\left\{x_{t} \in X ; t \in \boldsymbol{R}\right\}$, où $x_{-t}=-x_{t}$. En définisant $s x_{t}=x_{s t}(\forall s, \forall t \in R)$ et en induisant la topologie de $X$ sur $E, E$ est un espace vectoriel topologique sur $\boldsymbol{R}$. Si, pour deux nombres $t$ et $s$ de $\boldsymbol{R}$ avec $t \neq s, \alpha_{t}=\alpha_{s}$, alors $\alpha_{(t-s)}=\varepsilon$, et par suite $E$ est compact, mais cela est en contradiction avec l'hypothese que $N=\int_{0}^{\infty} \alpha_{t} d t$ est une mesure de Radon positive dans $X$. Par conséquent, $E$ et $R$ sont algébriquement isomorphe. Le semi-groupe $\left(\alpha_{t}\right)_{t \geqq 0}$ étant vaguement continu, l'application $t \rightarrow x_{t}$ est continue. Son inverse est évidemment continue, d'où la condition est nécessaire.

Montrons que la condition est suffisante. Soit $E$ l'espace vectoriel topologique sur $\boldsymbol{R}$ appartenant à $X$ et homéomorphe avec $\boldsymbol{R}$. Notons $x_{t}$ l'image de $t \in \boldsymbol{R}$ par l'application homéomorphe de $\boldsymbol{R}$ à $E$ et posons $\alpha_{t}=\varepsilon_{x_{t}}$; alors $\left(\alpha_{t}\right)_{t>0}$ est évidemment un semi-groupe vaguement continu. D'après l'hypothèse que $E$ et $\boldsymbol{R}$ sont homéomorphes, on a $x_{t} \rightarrow \infty$ dans $X$ avec $|\mathrm{t}| \rightarrow+\infty$. Par conséquent, $N=\int_{0}^{\infty} \alpha_{t} d t$ est un noyau de convolution de Hunt sur $X$. La fonction définie-négative associée au noyau $N$ est purement imaginaire, car $\alpha_{t} * \check{\alpha}_{t}=\varepsilon(\forall t \geqq 0)$.

Nous montrerons notre deuxième théorème suivant:

THÉORÈmE 2. Supposons qu'il existe un espace vectoriel topologique $E$ sur $\boldsymbol{R}$ appartenant $\grave{a} X$ et homéomorphe avec $\boldsymbol{R}$, et soit $\kappa$ un noyau de convolution non-zéro sur $R$ porté par $\boldsymbol{R}^{+}$. Alors pour que, quel que soit $N=\int_{0}^{\infty} \alpha_{t} d t$ un noyau de convolution de Hunt sur $X, N_{(x)}=\int \alpha_{t} d \kappa(t)$ ait un sens et soit un noyau de convolution de Hunt sur $X$, il faut et il suffit que $\kappa$ satisfasse an principe complet $d u$ maximum.

Démonstration. D'après le théorème 1 et le lemme 1 dans [9], la condition est suffisante. On montrera donc que la condition est nécessaire. Notons $x_{t}$ l'image de $t \in \boldsymbol{R}$ par l'application homéomorphe de $\boldsymbol{R}$ à $E$. D'après le présent lemme, $N=\int_{0}^{\infty} \varepsilon_{x_{t}} d t$ est un noyau de convolution de Hunt sur $X$, et donc $N_{(\kappa)}=\int \varepsilon_{x_{t}} d \kappa(t)$ est aussi un noyau de convolution de Hunt sur $X$, d'où $\kappa$ est un noyau de convolution de Hunt sur $\boldsymbol{R}$. Soit $\alpha$ une constante avec $0<\alpha<1$. Pour tout $t \geqq 0$, il existe une mesure de Radon positive $\nu_{t}$ dans $\boldsymbol{R}$ telle que la transformée de Fourier de $\nu_{t}$ soit égale à $\exp \left(-t|x|^{\alpha}\right)$. $\quad\left(\nu_{t}\right)_{t \geqq 0}$ est un semi-groupe vaguement continu sur $R$ et on a $\int_{0}^{\infty} \nu_{t} d t=C_{\alpha}|x|^{1-\alpha} d x$ dans $R$, où $C_{\alpha}$ est une 
constante positive. Posons $\tilde{\nu}_{t}=\int \varepsilon_{x_{s}} d \nu_{t}(s)$; Alors $\tilde{\nu}_{t}$ est une mesure de Radon positive dans $X$ portée par $E$ et $\left(\tilde{\nu}_{t}\right)_{t \geqq 0}$ est un semi-groupe vaguement continu. Evidemment $N_{\alpha}=\int_{0}^{\infty} \tilde{\nu}_{t} d t$ est un noyau de convolution de Hunt sur $X$. Il est symétrique et $\int d N_{\alpha}=+\infty$. D'après la proposition $2, \kappa$ doit être borné, d'où $\kappa$ satisfait au principe complet du maximum. La démonstration est ainsi complète.

Remarque. Sans la présente condition pour $X$, la présente équivalence n'a pas toujours lieu.

Nous considérerons ensuite une caractérisation d'un élément de la famille sous-ordonnée au noyau de convolution de Hunt donnée, en utilisant les fonctions définie-négatives.

Lemme 2. Soit $\kappa$ un noyau de convolution sur $\boldsymbol{R}$ porté par $\boldsymbol{R}^{+}$. Pour que $\kappa$ soit un noyau de convolution de Hunt borné sur $\boldsymbol{R}$, il faut et il suffit que la transformée de Laplace de $\kappa$ soit de la forme $\tilde{\kappa}=1 / F$ dans $(0,+\infty)$, où $F$ est une fonction de Bernstein.

On dit qu'une fonction non-négative et infiniment dérivable $F$ dans $(0,+\infty)$ est une fonction de Bernstein si $F^{(2 k)}(t) \leqq 0$ et $F^{(2 k-1)}(t) \geqq 0$ dans $(0,+\infty)(k=1,2, \cdots)$, où, pour un entier $m \geqq 0, F^{(m)}$ désigne la dérivée de $F$ d'ordre $m$. Il est connu qu'une fonction de Bernstein est de la forme

$$
F(t)=c_{1}+c_{2} t+\int(1-\exp (-t u)) d \sigma(u),
$$

où $c_{i}(i=1,2)$ et $\sigma$ sont respectivement une constante non-négative et une mesure de Radon positive dans $(0,+\infty)$ avec

$$
\int_{0}^{\infty} \frac{t}{1+t} d \sigma(t)<+\infty
$$

(cf. par exemple, [4]).

Montrons le lemme 2. Supposons d'abord que $\kappa$ est un noyau de convolution de Hunt borné sur $R$, et soit $\left(\kappa_{p}\right)_{p \geqq 0}$ la résolvante associée au noyau $\kappa$. Alors $p \int d \kappa_{p} \leqq 1(\forall p>0)$. On a, quel que soit $p>0, \tilde{\kappa}_{p}(t)<1 / p$ dans $(0,+\infty)$. En effet, si $\operatorname{supp}\left(\kappa_{p}\right) \neq\{0\}$, alors cela est évident. S'il existe un nombre $p>0$ tel que $\operatorname{supp}\left(\kappa_{p}\right)=\{0\}$, alors, quel que soit 
$q \geqq 0, \operatorname{supp}\left(\kappa_{q}\right)=\{0\}$, et par suite $q \int d \kappa_{q}<1$. D'après l'égalité

$$
\kappa=\frac{1}{p} \sum_{n=1}^{\infty}\left(p \kappa_{p}\right)^{n}, \quad \text { où } \quad\left(p \kappa_{p}\right)^{m}=\left(p \kappa_{p}\right)^{m-1} *\left(p \kappa_{p}\right) \quad(m \geqq 2),
$$

la transformée de Laplace $\tilde{\kappa}$ de $\kappa$ est une fonction positive et continue dans $(0,+\infty)$. On a, quels que soient $p>0$ et $q>0$,

$$
\frac{1-p \tilde{\kappa}_{p}}{\tilde{\kappa}_{p}}=\frac{1-q \tilde{\kappa}_{p}}{\tilde{\kappa}_{q}} .
$$

Notons $F$ cette fonction dans $(0,+\infty)$; alors $\tilde{\kappa}=1 / F$. La famille $\left(p\left(1-p \tilde{\kappa}_{p}\right)\right)_{p>0}$ converge uniformément vers $F$ sur tout compact de $(0,+\infty)$ avec $p \rightarrow+\infty$, car

$$
\tilde{\kappa}+\frac{1}{p}=\frac{1}{p\left(1-p \tilde{\kappa}_{p}\right)} .
$$

Donc $F$ est une fonction de Bernstein, d'où la condition est nécessaire.

Montron ensuite que la condition est suffisante. Pour cela, il suffit de voir que, quel que soit $p>0$, il existe un noyau de convolution sur $\boldsymbol{R}$ porté par $\boldsymbol{R}^{+}$tel que $\tilde{\kappa}_{p}=1 /(p+F)$, car si c'est vrai, $\left(\kappa_{p}\right)_{p \geqq 0}$ est évidemment la résolvante associée au noyau $\kappa$, où $\kappa_{0}=\kappa$. Écrivons

$$
F(t)=c_{1}+c_{2} t+\int(1-\exp (-t u)) d \sigma(u),
$$

où $c_{i}(i=1,2)$ et $\sigma$ sont respectivement une constante non-négative et une mesure de Radon positive dans $(0,+\infty)$ avec $\int \frac{t}{1+t} d \sigma(t)<+\infty$. Posons

$$
\nu_{n}=\frac{c_{2} n \varepsilon_{1 / n}+\sigma_{n}}{p+c_{1}+c_{2} n+\int d \sigma_{n}},
$$

où $\varepsilon_{1 / n}$ est la mesure de Dirac au point $1 / n$ dans $R$ et $\sigma_{n}$ est la restreinte de $\sigma$ sur $[1 / n,+\infty)$. Alors la suite

$$
\left(\left(p+c_{1}+c_{2} n+\int d \sigma_{n}\right)\left(1-\tilde{\nu}_{n}\right)\right)_{n=1}^{\infty}
$$

converge uniformément vers $p+F$ sur tout compact de $\boldsymbol{R}^{+}$avec $n \rightarrow+\infty$, et par suite la suite

$$
\left(\frac{1}{p+c_{1}+c_{2} n+d \sigma_{n}} \sum_{m=0}^{\infty}\left(\nu_{n}\right)^{m}\right)_{n=1}^{\infty}
$$


converge vaguement, où $\left(\nu_{n}\right)^{0}$ est la mesure de Dirac dans $\boldsymbol{R}$. Notons $\kappa_{p}$ sa limite; alors $\tilde{\kappa}_{p}=1 /(p+F)$, d'où la condition est suffisante.

THÉORÈme 3. Soient $N_{0}$ un noyau de convolution de Hunt borné sur $X$ et $\psi_{0}$ la fonction définie-négative associée au noyau $N_{0}$. Pour qu'un noyau de convolution $N$ sur $X$ appartienne à $H\left(N_{0} ; X\right)$, il faut et $i l$ suffit que $N$ soit un noyau de convolution de Hunt borné sur $X$ et que la fonction définie-négative associée au noyau $N$ soit de la forme $F\left(\psi_{0}\right)$, où $F$ est une fonction de Bernstein.

On remarque, d'après la représentation d'une fonction de Bernstein, qu'une fonction de Bernstein est considérée comme une fonction sur $\{z \in C ; \operatorname{Re} z \geqq 0\}$, où $C$ est le champ complexe.

Démonstration du théoème 3. Supposons d'abord que $N$ appartient à $H\left(N_{0} ; X\right)$. Alors il existe un noyau de convolution de Hunt borné sur $\boldsymbol{R}$ porté par $\boldsymbol{R}^{+}$tel que $N=\int \alpha_{t} d \kappa(t)$, où $\left(\alpha_{t}\right)_{t \geqq 0}$ est le semi-groupe associé au noyau $N_{0}$. Soit $\left(\kappa_{p}\right)_{p \geqq 0}$ la résolvante associée au noyau $\kappa$; alors, quel que soit $p>0, \iint d \alpha_{t} d \kappa_{p}(t) \leqq 1 / p$ et

$$
\int \hat{\alpha}_{t} d \kappa_{p}(t)=\int \exp \left(-t \psi_{0}\right) d \kappa_{p}(t)=\frac{1}{p+F\left(\psi_{0}\right)},
$$

où $F$ est une fonction de Bernstein telle que $\tilde{\kappa}=1 / F$ dans $(0,+\infty)$, et donc la fonction définie-négative associée au noyau $N$ est égale à $F\left(\psi_{0}\right)$.

Montrons ensuite que la condition est suffisante. De la même manière que dans le présent lemme, pour un nombre $p>0$ quelconque, il existe un noyau de convolution de Hunt borné $\kappa_{p}$ sur $\boldsymbol{R}$ porté par $\boldsymbol{R}^{+}$tel que $\tilde{\kappa}_{p}=1 /(p+F)$. Evidemment $\left(\kappa_{p}\right)_{p>0}$ est une résolvante. On a, quelle que soit $\varphi$ de $C_{K}^{+}(X)$,

$$
N * \varphi \geqq \int \alpha_{t} * \varphi d \kappa_{p}(t) \quad\left({ }^{\forall} p>0\right) .
$$

$\left(\alpha_{t}\right)_{t \geqq 0}$ étant semi-groupe et ayant $\alpha_{t} \rightarrow \varepsilon$ (vaguement) avec $t \rightarrow 0,\left(\kappa_{p}\right)_{p>0}$ est vaguement bornée. Par conséquent, il existe un noyau de convolution de Hunt borné $\kappa$ sur $R$ porté par $R^{+}$tel que $\tilde{\kappa}=1 / F$ dans $(0, \infty)$. Donc $N=\int \alpha_{t} d \kappa(t)$, d'où $N \in H\left(N_{0} ; X\right)$. La démonstration est ainsi complète. 


\section{La famille sous-ordonnée au noyau newtonien}

Dès maintenant, soit $X$ l'espace euclidien $\boldsymbol{R}^{n}$ à $n(\geqq 3)$ dimensions. Pour un point $x$ de $\boldsymbol{R}^{n}$, on note $|x|$ la distance entre $x$ et l'origine. On notera symboliquement $r^{2-n}$ le noyau newtonien $|x|^{2-n} d x$ sur $\boldsymbol{R}^{n}$. Rappelons d'abord le théorème de Schoenberg.

Proposition 3 (cf. par exemple, [3]). Soit $F(t)$ une fonction finie et continue sur $\boldsymbol{R}^{+}$. Pour que, quel que soit $n$ un entier $\geqq 1, F(|x|)$ soit de type positif dans $\boldsymbol{R}^{n}$, il faut et il suffit que $\boldsymbol{F}$ soit de la forme

$$
F(t)=\int \exp \left(-t^{2} u\right) d \mu(u)
$$

où $\mu$ est une mesure de Radon positive dans $\boldsymbol{R}^{+}$de masse totale finie.

La présente proposition porte naturellement la définition suivante.

DÉFINITION. Soient $\varphi$ une fonction finie et continue sur $\boldsymbol{R}^{+}$et $k$ un entier $>0$. On dit que $\varphi$ appartient à $D N_{k}$ si la fonction $\varphi(|x|)$ dans $\boldsymbol{R}^{k}$ est définie-négative. Si l'on a, quel que soit $k$ un entier positif, $\varphi \in D N_{k}$, alors on écrit $\varphi \in D N_{\infty}$.

Il est évident que si $k_{1} \leqq k_{2}, D N_{k_{1}} \supset D N_{k_{2}}$. Notre quatrième théorème est une caractérisation de la famille sous-ordonnée au noyau newtonien $r^{2-n}$.

THÉORغ̀me 4. Soit $N$ un noyau de convolution sur $\boldsymbol{R}^{n}$. Pour que $N$ appartienne à $H\left(r^{2-n} ; \boldsymbol{R}^{n}\right)$, il faut et il suffit que $N$ soit un noyau de convolution de Hunt sur $\boldsymbol{R}^{n}$ et qu'il existe une fonction $\varphi$ de $D N_{\infty}$ telle que la fonction définie-négative associée au noyau $N$ soit égale à $\varphi(|x|)$ sur $\boldsymbol{R}^{n}$.

Démonstration. Supposons d'abord $N \in H\left(r^{2-n} ; \boldsymbol{R}^{n}\right)$ et soit $\psi$ la fonction définie-négative associée au noyau $N$; alors, d'après le théorème 3 , il existe constantes $c_{1} \geqq 0, c_{2} \geqq 0$ et une mesure de Radon positive $\sigma$ dans $(0,+\infty)$ avec $\int \frac{t}{1+t} d \sigma(t)<+\infty$ telles que

$$
\psi(x)=c_{1}+c_{2}|x|^{2}+\int\left(1-\exp \left(-t|x|^{2}\right)\right) d \sigma(t),
$$

car il existe une constante $c>0$ telle que $\widehat{r^{-n}}=c|x|^{-2}$. Quel que soit $k$ un entier positif, la fonctions $|x|^{2}$ et $1-\exp \left(-t|x|^{2}\right)(\forall t \geqq 0)$ dans $\boldsymbol{R}^{k}$ 
sont définie-négatives, d'où la condition est nécessaire.

Montrons que la condition est suffisante. Pour tout entier $k>0$, la fonction $\varphi(|x|)$ est définie-négative dans $\boldsymbol{R}^{k}$, et par suite, quel que soit $p>0$, la fonction $1 /(p+\varphi(|x|))$ dans $\boldsymbol{R}^{k}$ est de type positif. D'après la proposition 3 , il existe une mesure de Radon positive $\kappa_{p}$ dans $\boldsymbol{R}^{+}$de masse totale finie telle que

$$
\frac{1}{p+\varphi(|x|)}=\int \exp \left(-t|x|^{2}\right) d \kappa_{p}(t)
$$

dans $\boldsymbol{R}^{k}$. On a, quels que soient $p>0$ et $q>0$,

$$
\begin{aligned}
& \int \exp (-t s) d \kappa_{p}(s)-\int \exp (-t s) d \kappa_{q}(s) \\
& =(q-p) \int \exp (-t s) d \kappa_{p}(s) \int \exp (-t s) d \kappa_{q}(s)
\end{aligned}
$$

pour tout $t$ de $\boldsymbol{R}^{+}$. D'après le fait que la transformation de Laplace est injective, on obtient que $\left(\kappa_{p}\right)_{p>0}$ est une résolvante de noyau de convolution sur $\boldsymbol{R}$. De la même manière que dans le théorème 3 , on peut montrer $N \in H\left(r^{2-n} ; \boldsymbol{R}^{n}\right)$. La démonstration est ainsi complète.

\section{BIBLIOGRAPHIES}

[1] J. Deny: Sur l'équation de convolution $\mu=\mu * \sigma$. Sém. B-C-D, 4ème année, 1959/60, no. 5 .

[2] - : Noyaux de convolution de Hunt et noyaux associés à une famille fondamentale. Ann. Inst. Fourier, 12, 1962, 643-667.

[3] W. F. Donoghue, Jr: Distributions and Fourier transforms. Acad. press, New York, 1969.

[4] C. S. Herz: Analyse harmonique à plusieurs variables. Sém. Math. d'Orsay, 1965/66.

[5] I. Higuchi et M. Itô: Characterization of the relative domination principle. Nagoya Math. J., 50, 1973, 175-184.

[6] M. Itô: Sur les principes divers du maximum et le type positif. Nagoya Math. J., 44, 1971, 133-164.

[7] - Sur le principe de domination pour les noyaux de convolution. Nagoya Math. J., 50, 1973, 149-173.

[8] - Caractérisation du principe de domination pour les noyaux de convolution, à paraître.

[9] — : Sur la famille sous-ordonnée au noyau de convolution de Hunt, Nagoya Math. J., 51, 1973, 45-56.

[10] M. Kishi: Puissance fractionnaire d'un noyau positif dont le carré est encore un noyau. Nagoya Math. J., 44, 1971, 79-88.

\section{Université de Nagoya}

